\title{
Study on Fluorescence Spectra of B Vitamins
}

\author{
Yang Hui ${ }^{1, a}$, Xiao Xue ${ }^{2, b}$, Hu Lan ${ }^{1, c}$ and Zhibin Hou ${ }^{1, d}$ \\ ${ }^{1}$ New Star Application Technology Institute, Huangshan Road, Hefei, 230031, China \\ ${ }^{2}$ Key Lab of Environmental Optics \& Technology, AIOFM, CAS, \\ Shushan Hu Road, Hefei, 230031, China

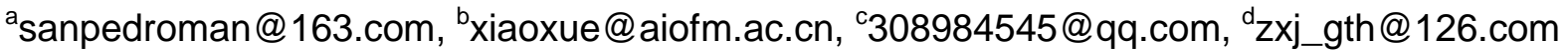

\begin{abstract}
Keywords: Thiamine/Vitamin B1, Riboflavin/Vitamin B2; Pyridoxine/Vitamin B6, Fluorescence Spectra, Compound Vitamin B, PARAFAC.
\end{abstract}

\begin{abstract}
This paper presents the intrinsic fluorescence characteristics of vitamin B1, B2 and B6 measured with 3D fluorescence Spectrophotometer. Three strong fluorescence areas of vitamin B2 locate at $\lambda \mathrm{ex} / \lambda \mathrm{em}=270 / 525 \mathrm{~nm}, 370 / 525 \mathrm{~nm}$ and $450 / 525 \mathrm{~nm}$, one fluorescence area of vitamin B1 locates at $\lambda \mathrm{ex} / \lambda \mathrm{em}=370 / 460 \mathrm{~nm}$, two fluorescence areas of vitamin B6 locate at $\lambda \mathrm{ex} / \lambda \mathrm{em}=250 / 370 \mathrm{~nm}$ and 325/370nm were found. Using the PARAFAC algorithm, 10 vitamin B1, B2 and B6 mixed solutions were successfully decomposed, the emission profiles, excitation profiles, central wavelengths and the concentration of the three components were retrieved precisely through about 5 iteration times. Vitamin B1, B2 and B6 components were found in two compound vitamin B samples, the fluorescence contribution of pyridoxine is weakest in both samples, and the concentration of riboflavin, and thiamine are $0.11 \mathrm{mg} / 1,0.32 \mathrm{mg} / 1$ respectively for sample $1^{\text {st }}$, and $0.12 \mathrm{mg} / \mathrm{L}$ for sample $2^{\text {nd }}$.
\end{abstract}

\section{Introduction}

$\mathrm{B}$ vitamins are water-soluble and of great importance for the health of people, they can not be synthesized within the body, but be obtained from daily food, so the content measurement of B vitamins in the food is necessary for food security and research. Riboflavin, known as vitamin B2, is required for a wide variety of cellular processes, and plays a key role in energy metabolism, and is required for the metabolism of fats, ketone bodies, carbohydrates, and proteins [1]. Thiamine, known as vitamin B1, is used in many different body functions and deficiencies may have far reaching effects on the body, yet very little of this vitamin is stored in the body, and depletion of this vitamin can happen within 14 days. Vitamin B6 can be found in a variety of forms in the foods we eat as well as in our bodies, it is involved at several steps in the metabolism of carbohydrates, is one of several B vitamins required for proper production of messaging molecules in our nervous system and brain.

PARAFAC, a three way-decomposition method, has been found to be very useful in identifying the independent spectra of different types of fluorophores [2]. Compared to its predecessor, Principal Component Analysis (PCA) technique, PARAFAC provides both a quantitative and qualitative model of the data and separates the complex signal measured into its individual underlying fluorescent phenomena with specific excitation and emission spectra. It can track even small variations in EEM datasets by separating several independent groups of fluorophores from the overlapped components with a high resolution, so it is commonly used technique to monitor the mixed fluorescence EEMs. On the other hand, the weakness of PARAFAC model may include the assumption of the independence among the estimated components in the model, and potential inclusion of one or more poorly estimated components, which may significantly affect the spectra and scores of all other components [3].

Accordingly, in this paper, we discussed not only the fluorescence spectra of riboflavin and thiamine individually, but also the overlap of fluorescence spectra of vitamin B1, vitamin B2 and vitamin B6 within the Ex/Em area with wavelength of $200 \sim 550 \mathrm{~nm} / 370 \sim 610 \mathrm{~nm}$, and by means of Parallel factor analysis (PARAFAC) method. 


\section{Experimental Section}

Instruments and Reagents. The Molecular $\Sigma \mathrm{H}_{2} \mathrm{O}$ ultra pure water machine (Shanghai Molecular Co. Ltd) was used to generate the ultra purified water, UPW whose $\mathrm{pH}$ value is 5.4. The vitamin B2 mother liquid were compounded with Riboflavin from Amresco co.Ltd whose purity greater than or equal to $98 \%$, the vitamin B1 from Sigma co.Ltd with purity greater than or equal to $99 \%$, and the vitamin B6 from Sigma co.Ltd with purity greater than or equal to $98 \%$. All reagents and materials were weighed with Mettler Toledo precise electronic balance. The solvents used in the experiments include $0.2 \mathrm{~mol} / \mathrm{L} \mathrm{Na}_{2} \mathrm{HPO}_{4}$ buffer, $0.1 \mathrm{~mol} / \mathrm{L} \mathrm{C}_{6} \mathrm{H}_{8} \mathrm{O}_{7} \cdot \mathrm{H}_{2} \mathrm{O}$ buffer, $\mathrm{Na}_{2} \mathrm{HPO}_{4}$ and $\mathrm{C}_{6} \mathrm{H}_{8} \mathrm{O}_{7} \cdot \mathrm{H}_{2} \mathrm{O}$ mixed buffer, $1 \% \mathrm{~K}_{3}\left[\mathrm{Fe}(\mathrm{CN})_{6}\right]$ buffer, $2 \mathrm{~mol} / \mathrm{L} \mathrm{NaOH}$ buffer and $3 \mathrm{~mol} / \mathrm{L} \mathrm{H}_{2} \mathrm{SO}_{4}$ buffer.

The vitamin B2, B1 and B6 mother liquid concentration are 20, 10 and 24mg/L respectively. The mother reagent solutions were transferred through Dragon Lab whole disinfection manual single channel adjustable liquid shifter and dilute to working solutions of different concentrations. All reagents were of analytical grade, all solutions and put in amber glass bottles and stored in a refrigerator $\left(4^{\circ} \mathrm{C}\right)$ because of the light sensitivity of vitamin $\mathrm{B} 2$.

3D fluorescence intensity measurements were carried out on an F-7000 FL spectrophotometer (Hitachi High-Technologies Corporation, Japan).

Instrument Settings and Experiment Procedure. For the fluorescence EEM measurements of vitamin B2, the spectrophotometer excitation wavelength ranged from $200.0 \mathrm{~nm}$ to $550.0 \mathrm{~nm}$, emission wavelength ranged from $450.0 \mathrm{~nm}$ to $650.0 \mathrm{~nm}$, scan speed was set at $12000 \mathrm{~nm} / \mathrm{min}$ with excitation and emission sampling interval of $10.0 \mathrm{~nm}$, excitation and emission slit of 5.0nm, the PMT voltage was set at $700 \mathrm{~V}$. And for thiamine, the excitation wavelength ranged from $300.0 \mathrm{~nm}$ to $400.0 \mathrm{~nm}$, emission wavelength ranged from $350.0 \mathrm{~nm}$ to $550.0 \mathrm{~nm}$. And for pyridoxine, the excitation/emission wavelength range is $200.0 \sim 350.0 \mathrm{~nm} / 300.0 \sim 450.0 \mathrm{~nm}$, and accordingly, for fluorescence EEM of vitamin mixed solution, the excitation/emission wavelength range is $200.0 \sim 500.0 \mathrm{~nm} / 350.0 \sim 650.0 \mathrm{~nm}$. All experiments were performed at room temperature at $25^{\circ} \mathrm{C}$.

The $1^{\text {st }}$ level and $2^{\text {nd }}$ level Rayleigh scattering, Raman scattering and other background components within the fluorescence signals were corrected for the following analysis.

PARAFAC Method for Multi-components Discrimination. Based on the tri-linear decomposition theory, the parallel factor analysis(PARAFAC) method is a kind of mathematical model implemented through alternating least squares algorithm, which is widely applied to analyze three-dimensional or multi-dimensional data, to decompose $N$-dimensional data to the $N$ load matrixes.

The measured fluorescence spectrum EEM data is a $I \times J \times K$ matrix, in which, $I$ indicates the number of the samples, while $J$ and $K$ are the number of excitation wavelengths and emission wavelengths of samples respectively. Using parallel factor decomposition model, the fluorescence spectrum data matrix can be decomposed to score matrix $A$, load matrix $B$ and $C$. The decomposition model can be represented as

$$
x_{i j k}=\sum_{f=1}^{F} \alpha_{i f} b_{j f} c_{k f}+\varepsilon_{i j k}, i=1,2, \ldots, I, j=1,2, \ldots, J, k=1,2, \ldots, K
$$

where, $\mathrm{x}_{\mathrm{ijk}}$ is the fluorescence intensity of sample $i$ at excitation wavelength $j$ and emission wavelength $k, F$ is the column number of load matrix, or the number of factors, $\varepsilon_{i j k}$ is the residual element, $\alpha_{i f}, b_{j f}, c_{k f}$ are the elements in load matrix A, B and $\mathrm{C}$ respectively. The algorithm will be aborted until convergence of the PARAFAC model, that is, the minimum loss function

$$
f_{S S R}=\sum_{i=1}^{I} \sum_{j=1}^{J} \sum_{k=1}^{K} e^{2}{ }_{i j k}<10^{-6}
$$

In this study, PARAFAC modeling was performed using the MATLAB 7.0 code. 


\section{Fluorescence EEM Characteristics of Riboflavin, Thiamine and Pyridoxine}

For riboflavin/vitamin B2, there are three strong fluorescence areas, whose center locate at $\lambda \mathrm{ex} / \lambda \mathrm{em}=270 / 525 \mathrm{~nm}, 370 / 525 \mathrm{~nm}$ and $450 / 525 \mathrm{~nm}$ respectively, and the emission wavelength ranges from about $500 \mathrm{~nm}$ to $600 \mathrm{~nm}$. As for thiamine/vitamin B1, it has only one strong fluorescence areas, whose center locate at $\lambda \mathrm{ex} / \lambda \mathrm{em}=370 / 460 \mathrm{~nm}$, the excitation wavelength ranges from about $320 \mathrm{~nm}$ to $400 \mathrm{~nm}$, and the emission wavelength ranges from about $370 \mathrm{~nm}$ to $550 \mathrm{~nm}$. Accordingly, as for pyridoxine/bitamin B6, there are two strong fluorescence areas, whose center locate at $\lambda \mathrm{ex} / \lambda \mathrm{em}=240 / 370 \mathrm{~nm}$ and $320 / 370 \mathrm{~nm}$ respectively, and the emission wavelength ranges from about $330 \mathrm{~nm}$ to $440 \mathrm{~nm}$.
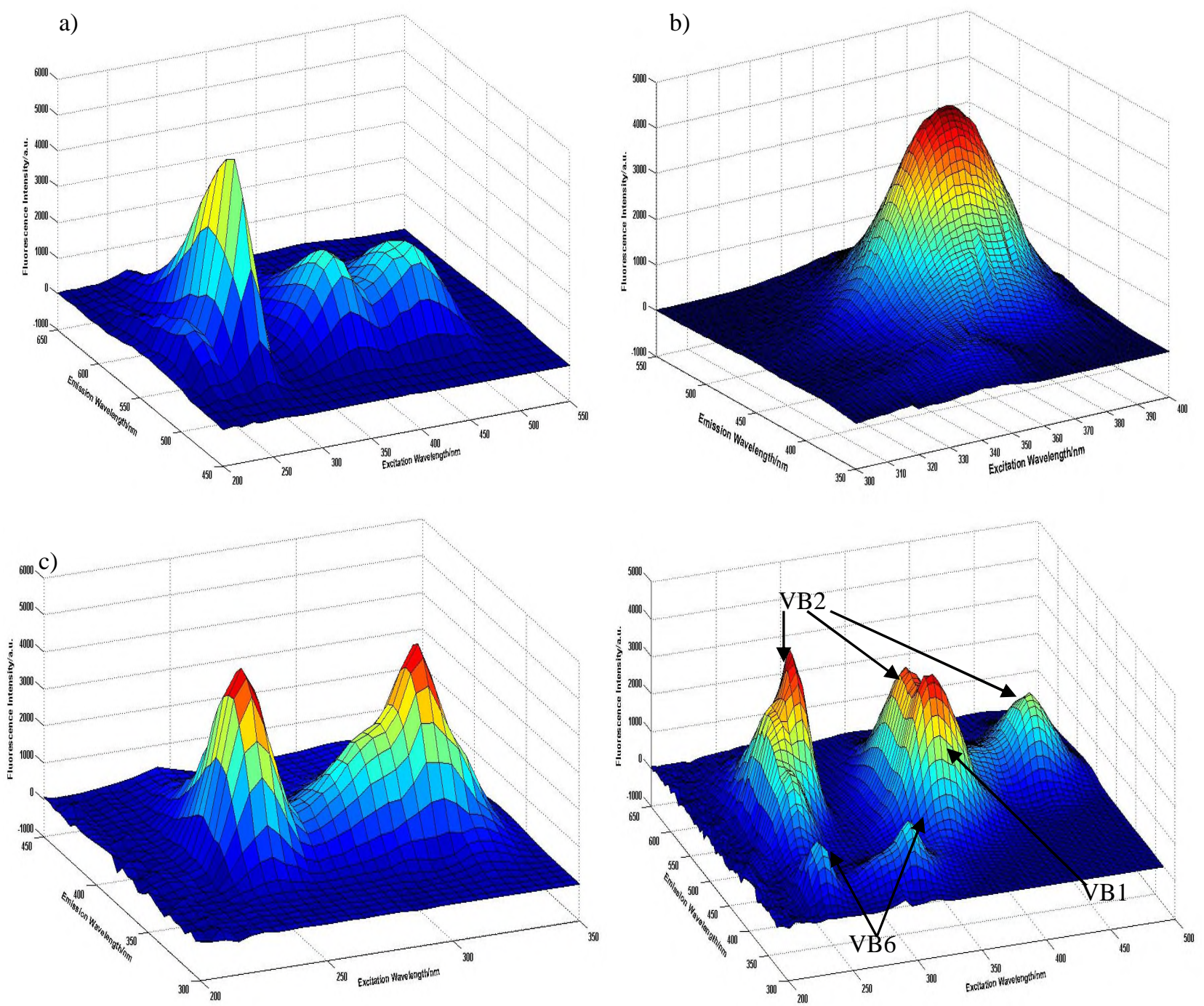

Fig. 1 Fluorescence intensity distribution of riboflavin@200ug/L, thiamine@75ug/L, pyridoxine@200ug/L, and their mixture.

So, according to the mirror symmetry of the excitation and emission spectra, it can be concluded that, the main excitation/emission wavelength of riboflavin, thiamine and pyridoxine locate at $\lambda \mathrm{ex} / \lambda \mathrm{em}=450 / 525 \mathrm{~nm}, 370 / 460 \mathrm{~nm}$ and $320 / 370 \mathrm{~nm}$ respectively, the overlapping of these three $3 \mathrm{D}$ fluorescence spectra is obvious. 


\section{Analysis of Fluorescence Spectra of 3-Components Mixed Solutions}

The fluorescence spectra of 10 solutions mixed with thiamine, riboflavin and pyridoxine of different concentration were measured and decomposed by PARAFAC method, the retrieved excitation and emission profiles were shown in Fig. 2.
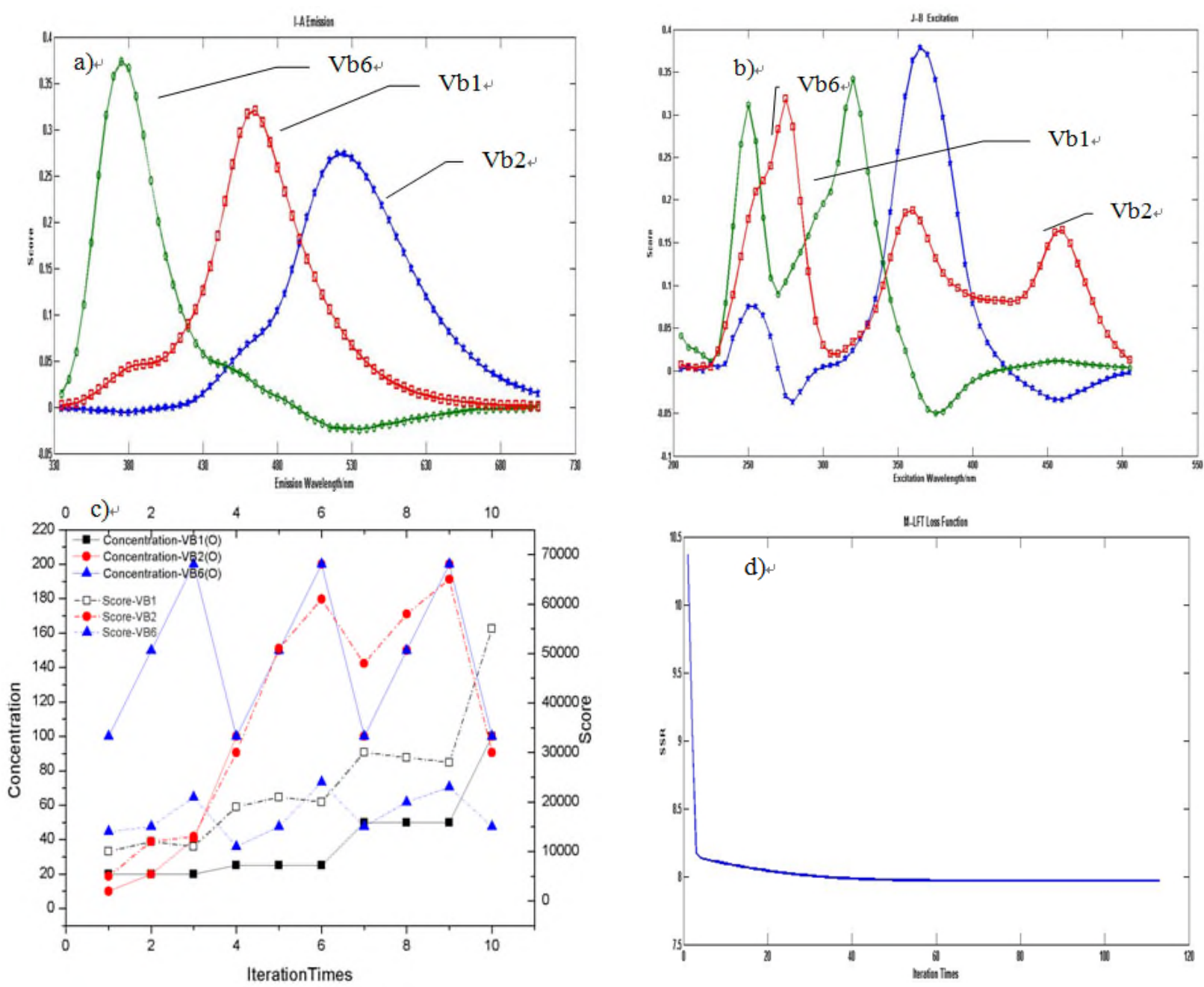

Fig. 2 Excitation and emission spectral profile retrieved by PARAFAC algorithm, (a)Emission spectra, (b) Excitation spectra, (c) original and retrieved concentrations, (d) SSR and iteration number.

The emission/excitation spectra retrieved by PARAFAC are shown in Fig. 2(a) and (b). From Fig. 2(a) and Fig. 1, it can be seen that the retrieved emission profile and central wavelength of riboflavin, thiamine and pyridoxine are coincident with their real emission profiles very well, the retrieved excitation profile and central wavelength of riboflavin and pyridoxine are coincident with their real excitation profiles. But as for the retrieved excitation profile and central wavelength of pyridoxine, there exists an extra weak excitation peak at $250 \mathrm{~nm}$.

The good linear correlations of original and retrieved concentrations of thiamine, riboflavin and pyridoxine are shown in Fig. 2(c). From Fig. 2(d) one can see that SSR of PARAFAC logarithms decreases quickly and sharply at the beginning of the iteration times, the SSR is stable when iteration times $\geq 5$. 
Table 1. Original and analytical concentrations of riboflavin, thiamine and pyridoxine.

\begin{tabular}{|l|c|c|c|c|c|c|}
\hline \multirow{3}{*}{ Sample } & \multicolumn{6}{|c|}{ Component } \\
\cline { 2 - 7 } & \multicolumn{2}{|c|}{ Thiamine } & \multicolumn{2}{c|}{ Riboflavin } & \multicolumn{2}{c|}{ Pyridoxine } \\
\cline { 2 - 7 } & $\begin{array}{c}\text { Original } \\
\text { (ug/L) }\end{array}$ & $\begin{array}{c}\text { Retrieved } \\
\text { score }\end{array}$ & $\begin{array}{c}\text { Original } \\
(\mathbf{u g} / \mathbf{L})\end{array}$ & $\begin{array}{c}\text { Retrieved } \\
\text { score }\end{array}$ & $\begin{array}{c}\text { Original } \\
(\mathbf{u g} / \mathbf{L})\end{array}$ & $\begin{array}{c}\text { Retrieved } \\
\text { score }\end{array}$ \\
\hline a) & 20 & 10000 & 10 & 1000 & 100 & 14000 \\
\hline b) & 20 & 12000 & 20 & 1200 & 150 & 15000 \\
\hline c) & 20 & 15000 & 40 & 1300 & 200 & 21000 \\
\hline d) & 25 & 29000 & 100 & 50000 & 100 & 11000 \\
\hline e) & 25 & 31000 & 150 & 53000 & 150 & 15000 \\
\hline f) & 25 & 25000 & 200 & 51000 & 200 & 14000 \\
\hline g) & 50 & 30000 & 100 & 48000 & 100 & 15000 \\
\hline h) & 50 & 29000 & 150 & 47000 & 150 & 20000 \\
\hline i) & 50 & 28000 & 200 & 45000 & 200 & 23000 \\
\hline j) & 100 & 55000 & 100 & 30000 & 100 & 15000 \\
\hline
\end{tabular}

\section{Analysis of Fluorescence Spectra of Two Compound Vitamin B Samples}
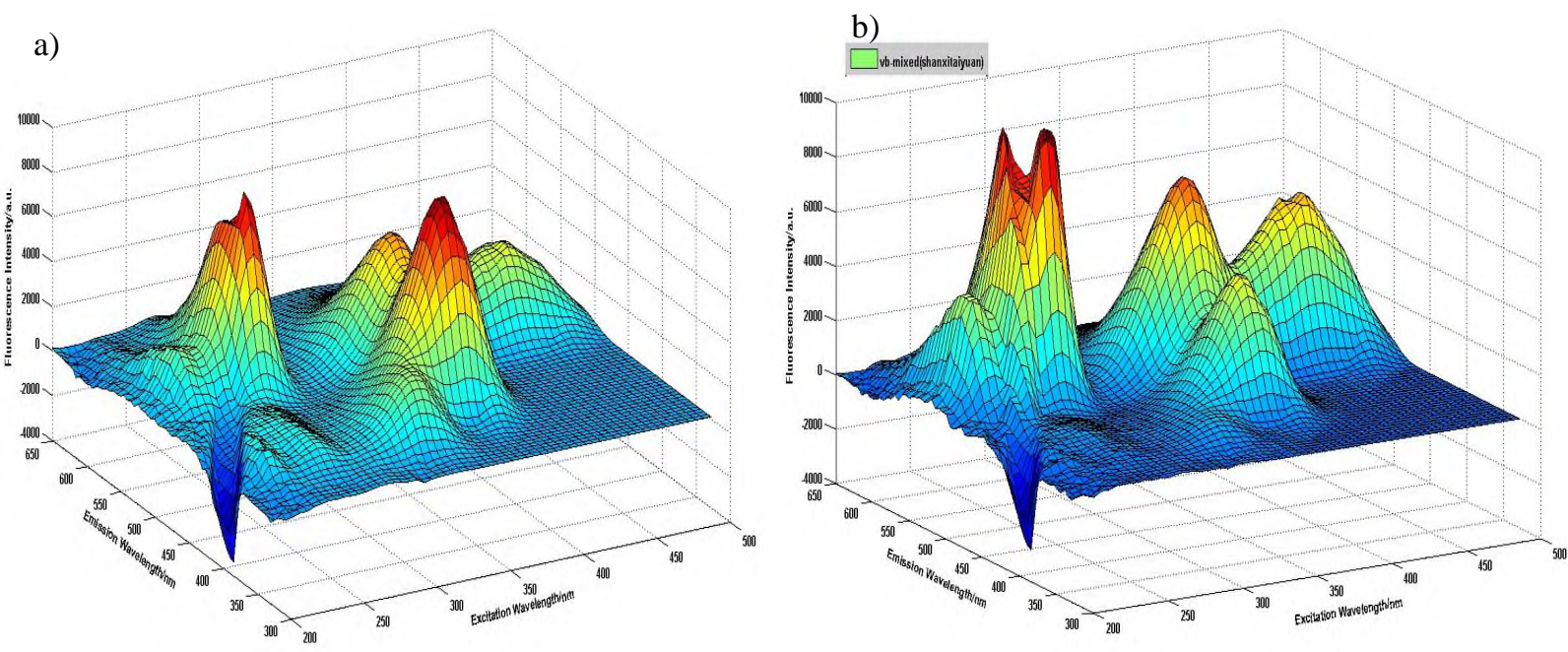

Fig. 3 Fluorescence intensity distributions of two samples.

The $3 \mathrm{~d}$ fluorescence spectra of 2 compound vitamin B samples were shown in Fig. 3. The $1^{\text {st }}$ sample, compound vitamin B injection, is from Shanxi Ruichenkelong veterinary medicine Co.Ltd, and the $2^{\text {nd }}$ sample, compound vitamin B tablets is produced by Shanxi Tianyuan medical limited. From Fig. 3, it can be seen that: 1) the fluorescence intensity of three kinds of vitamins are obvious in two samples, 2)in sample $1^{\text {st }}$, the component of thiamine is the highest one, but in sample $2^{\text {nd }}$, the major component is riboflavin, 3) in both samples, the contribution of pyridoxine are the weakest. 4) the concentration of riboflavin, and thiamine are $0.11 \mathrm{mg} / 1,0.32 \mathrm{mg} / 1$ respectively for sample $1^{\text {st }}$, and $0.12 \mathrm{mg} / \mathrm{L}$ for sample $2^{\text {nd }}$.

\section{Conclusions}

Using fluorescence Spectrophotometer, the intrinsic fluorescent characteristics of vitamin B1, B2 and B6 were measured with solutions of different concentration and the effect of $\mathrm{pH}$ to fluorescent profile was also discussed. Vitamin B1, B2 and B6 mixed solutions are successfully decomposed and resolved by PARAFAC algorithm. The retrieved emission profiles, excitation profiles, central wavelengths and the concentration of three components are coincident precisely with real emission profiles, excitation profiles, and central wavelengths of each component. 


\section{Acknowledgments}

The work was supported by the National Natural Science Foundation of China named "Study on Technology of Ultraviolet Laser-induced Fluorescence LIDAR for Bioagent Remote Sensing" No. 41375026.

\section{References}

[1] National Academy of Sciences, Sensor Systems for Biological Agent Attacks: Protecting Buildings and Military Bases, Committee on Materials and Manufacturing Processes for Advanced Sensors, Board on Manufacturing and Engineering Design, Division on Engineering and Physical Sciences, National Academies Press Washington URL: http://www.national-academies.edu/bmed, (2004).

[2] C. A. Stedmon, S. Markager and R. Bro, Tracing dissolved organic matter in aquatic environments using a new approach to fluorescence spectroscopy, Mar. Chem. 82 (2003) 239-254.

[3] K. R. Murphy, A. Hambly, S. Singh, R. K. Henderson, A. Baker, R. Stuetz and S. J. Khan, Organic matter fluorescence in municipal water recycling schemes: Toward a unified PARAFAC model, Environ. Sci. Technol., 45 (2011) 2909-2916. 